\title{
AHR los cuerpos FICTICIOS DE LA www.aibr.org \\ BIOMEDICINA. \\ EL \\ PROCESO \\ DE \\ CONSTRUCCIÓN DEL GÉNERO EN LOS PROTOCOLOS MÉDICOS DE ASIGNACIÓN DE SEXO EN BEBÉS INTERSEXUALES
}

\section{Nuria Gregori Flor}

Investigadora predoctoral, Universidad de Valencia (España). E-mail: nugreflor@hotmail.com

\begin{abstract}
Resumen
El nacimiento de un bebé del cual no se puede decir si es niña o niño con una simple inspección visual, supone la activación de una serie de resortes médicos y tecnológicos dirigidos a desvelar qué se oculta detrás de tal indefinición genital. Todos los esfuerzos dedicados a elaborar categorías, definiciones, criterios y protocolos para diagnosticar tal ambigüedad, así como el auge en investigación y desarrollo de nuevas tecnologías biomédicas para corregirla, revelan la inquietud que provoca dentro de nuestro orden sociocultural la ambigüedad y lo inclasificable en relación al sexo, al género y a la sexualidad. Los mandatos de un orden "monosexual" -la idea de que una persona sólo puede tener un único sexo/género y que este es estable para toda la vida- y del conocido como "dimorfismo sexual" -la creencia o convicción dominante en Occidente de la existencia de tan sólo dos sexos posibles, mujer y varón- obliga a confeccionar cuerpos ficticios que eliminan la verdadera variabilidad sexual humana. Será la institución médica y los profesionales médicos responsables de estos recién nacidos, los encargados de buscar qué sexo "verdadero" se esconde detrás de tanta ambigüedad, erigiéndose como responsables del proceso de sexuación en nuestra sociedad.
\end{abstract}

\section{Palabras clave}

Intersexualidad, asignación de sexo, identidad sexual/de género, proceso de sexuación, medicalización.

\begin{abstract}
After the birth of a child, when it is not possible to determinate whether it is a girl or a boy by simple visual inspection, the application of different medical technologies is required, in order to unveil what lies behind the genital uncertainty. All the efforts to create categories, definitions, criteria and protocols to define such ambiguity, and the boom of investigation with new biomedical technologies to correct it, reveal the anxiety provoked, in our socio-cultural order, by this ambiguity and the unclassifiable in relation to sex, gender and sexuality. The rules of our "monosexual" order -the idea that a person can only have one single sex/gender which is stable throughout its whole life- and of what is know as "sexual dimorphism" -the dominant belief or conviction, in the western world, that only two possible sexes, female and male, exist- force the creation of fictitious bodies which eliminate the real human sexual variability. Thus, medical institutions and, specifically, the physicians in charge of the newborn
\end{abstract}


children have the responsibility to find out the "real" sex hidden behind this ambiguity, establishing themselves as the ones responsible for the sexuation process in our society.

\section{Key words}

Intersexuality, sex assignment, sexual/gender identity, sexuation process, medicalization.

\section{Introducción}

"La verdad biológica es, rara vez, una verdad completa y estable, sino fragmentaria y provisional". Gregorio Marañón, 1930

$E^{\prime}$ siguiente artículo hace un recorrido analítico alrededor de la etiqueta diagnóstica o nosológica de "intersexualidad" o de "estados intersexuales". Concretamente sobre los discursos médicos que nombran, definen y clasifican la intersexualidad, sobre los protocolos de diagnóstico y tratamiento de los denominados por la biomedicina bebés "intersexo", así como las prácticas médicas sobre cuerpos "intersexo". La información ha sido obtenida de manuales, artículos y revistas de divulgación científica especializados en endocrinología, ginecología, genética, urología y cirugía, y también de las entrevistas realizadas a profesionales médicos - de las especialidades de ginecología, pediatría endocrinológica, urología y cirugía- que trabajan actualmente en instituciones sanitarias españolas hospitales, centros de salud y clínicas de carácter público o privado-, diagnosticando, y por tanto planificando acciones sobre personas que sitúan bajo la etiqueta de algún síndrome intersexual.

El foco de atención de este artículo se va situar en el ámbito de los presupuestos médicos sobre sexo, género y sexualidad presentes de forma más o menos explícita en los discursos y prácticas médicas existentes en los protocolos de diagnóstico y tratamiento de bebés intersexuales.

La historia del cuerpo intersexual, anteriormente conocido como hermafrodita, es la historia de un cuerpo vigilado, castigado y regulado, tal como indica Foucault (1998), por el hecho de ser diferente o inusual. En Vigilar y Castigar (1998), el autor explica cómo han cambiado las formas de castigo, pasando de una regulación del cuerpo más directa, cruenta y represiva para volverse, en manos de las instituciones, en concreto de la institución médica, en una forma de castigo más sofisticada e indirecta. Así, el cuerpo sexuado es sometido a esta nueva forma de poder que Foucault bautizó como "biopoder" incluso antes del nacimiento. El proceso de segregación entre "normales" y "anormales", o lo que es lo mismo, entre "sanos" y "enfermos" o "patológicos", comienza ya en el vientre materno mediante las técnicas de diagnóstico prenatal. Es difícil escapar al cerco creado por los circuitos institucionales médicos en los primeros años de vida: el seguimiento continúa en la sala de paritorio de cualquier hospital y con las revisiones periódicas realizadas en los servicios de pediatría de los centros de salud donde los programas de salud se preocupan por clasificar a los niños dentro de percentiles marcados para cada edad y sexo, de hacer cumplir el calendario vacunal vigente y de detectar cualquier "anomalía" o "problema" que pudiera aparecer. El diagnóstico está asegurado. Nadie es capaz de burlar la vigilancia institucional sobre el cuerpo en nuestra sociedad. 
En este sentido, el cuerpo sexuado es blanco seguro del aparato biomédico. La institución homologada para legitimar el sexo de los individuos en nuestra sociedad es la institución médica y los profesionales médicos que trabajan en ella, y en consecuencia, los discursos que surgen de ella son cada vez más sociales e impregnan cada día más el imaginario social sobre el cuerpo, el sexo y la sexualidad.

\section{¿Cómo y por qué somos intersexuales?}

"Las fantasmagorías de la naturaleza pueden promover los extravíos del libertinaje" Foucault (1985)

En cualquier definición de intersexualidad o de un estado intersexual de un manual o tratado de endocrinología pediátrica (Behrman, 1990; Argente, 2000), de ginecología (Botella Llusiá, 1993; Calaf, 2000) o de genética (Thomson, 2002), así como en las obtenidas en las entrevistas realizadas, va precedida de una explicación sobre cuál es el proceso normal de diferenciación sexual en los humanos y qué debemos entender como sexo biológico o constitucional. Siguiendo a Audí Parera (2002), la biomedicina ha distinguido clásicamente tres etapas o niveles de diferenciación sexual: el sexo genético -células $46 \mathrm{XX}$ o $46 \mathrm{XY}$-, el sexo gonadal -ovarios o testículos- y el sexo genital vagina, vulva, pene, próstata- que quedan determinados en el periodo fetal. Durante la infancia, pero sobre todo durante la pubertad y en el adulto, hay que añadir el sexo fenotípico -caracteres sexuales secundarios-, el psicosexual y el sexo social.

En este proceso fisiológico intervienen multitud de factores, enzimas y hormonas que como resultado de continuas reacciones químicas definirán sexualmente el nuevo ser. La forma en que la biomedicina explica este proceso nos remite a un delicado mecanismo de extremada complejidad en el cual cualquier fallo, por mínimo que sea, puede romper la cadena "normal" de formación de un supuesto dimorfismo sexual en humanos y aparecer un estado intersexual. Por ejemplo, dentro de esta lógica explicativa del proceso de sexuación humana, Calaf (2000) expone cómo las alteraciones producidas en el proceso de diferenciación sexual normal, a partir del sexo genético, pasando por el gonadal, al sexo genital o fenotípico, producen estados intersexuales o intersexos. Es decir, situaciones patológicas en las que el aparato genital externo evoluciona tan sólo de forma parcial hacia una diferenciación de tipo masculino, haciéndose difícil la atribución de un sexo social determinado (2000: 67). Piró Biosca por su parte, define los estados intersexuales como "resultado de errores en el desarrollo del feto, ya sea a nivel cromosómico, embriológico o por defectos bioquímicos" (2002: 129) y Gracia Bouthelier como "consecuencia de diversas alteraciones en la compleja fisiología que da origen a una buena formación tanto de genitales internos como externos" (2002: 83). Según esto, cualquier combinación posible que no responda a la secuencia prevista de diferenciación sexual entra en la esfera de lo patológico, de lo alterado o de lo erróneo.

De los discursos obtenidos a partir de las entrevistas a profesionales médicos se extrae que en muchas ocasiones, en la práctica clínica cotidiana, se empieza a hablar de intersexualidad 
simplemente cuando aparecen unos genitales que no responden a las expectativas morfológicas establecidas culturalmente para mujeres y para varones:

Una situación intersexual es aquel sujeto que nace y tú no puedes definir si es chico o chica o que hay razones para que tú no puedas, simplemente por los genitales externos, definirte hacia un lado o hacia otro. Eso ya a mí me parece suficiente como para definir una situación intersexual (Entrevista a NR, Enero de 2005).

En última instancia, la apariencia genital externa puede ser suficiente para hablar de intersexualidad, independientemente de que exista o no disfunción orgánica. La variabilidad genital en este caso se problematiza, y en consecuencia, se medicaliza. En cualquier caso, la explicación biomédica del proceso normal de diferenciación sexual y de los problemas o errores surgidos en esta secuencia, transforma la variabilidad sexual humana en estadios patológicos de ambigüedad genital producto de errores en el desarrollo ${ }^{1}$.

\title{
Protocolos médicos de determinación del sexo definitivo
}

\author{
"AC- ¿Es algo grave doctor? \\ $D-$ No, no, en absoluto. Aunque habrá que intervenir, desde luego. \\ AC- ¿Intervenir? \\ $D$ - Si, pero no se alarme; será muy sencillo. \\ AC- Perdone doctor, pero yo físicamente me encuentro muy bien. No veo la \\ relación que... \\ $D$ - Pues la hay. Claro que la hay. No se puede tener la mente enferma sin que \\ el cuerpo lo este también. $Y$ no se trata precisamente de una dependencia o \\ interrelación, sino de una completa y total identidad. \\ AC- Le agradeceré que me hable sin rodeos. Yo soy una mujer fuerte, $y$ \\ valiente. \\ $D$ - Ya lo sé. Y tiene razón en todo, excepto en una cosa: es usted fuerte y \\ valiente, pero no es usted una mujer, no lo es." \\ Jaime de Armiñan (1971) "Mi querida señorita".
}

Podemos asegurar, que con anterioridad al trabajo del polémico pediatra psicoendocrinólogo John Money, no existían protocolos para el tratamiento de la intersexualidad tal y como es entendida desde la práctica médica actual. Sus protocolos surgieron en la década de los cincuenta partiendo de la teoría en la cual defendía que la identidad de género ${ }^{2}$ es neutral en el nacimiento y en la infancia temprana y que posteriormente se determina por los genitales y la crianza. Dicho de otro modo, que la identidad de género sería exclusivamente el producto de la crianza y de la socialización. Su tesis titulada Hermafroditismo: Una Investigación en la Naturaleza de una Paradoja Humana (1952) trataba sobre lo que él consideraba "experimentos de la naturaleza", refiriéndose a los individuos que investigó como "individuos nacidos con un defecto congénito en sus órganos sexuales" (Money, 1982: 5). Para el autor, el hermafroditismo significa que un niño nace con su anatomía sexual impropiamente diferenciada, es decir, que está sexualmente inacabado (Money, 1982: 24).

\footnotetext{
${ }^{1}$ Los estados o síndromes intersexuales que surgieron de forma más recurrentes en las entrevistas son diagnósticos de "Síndrome de insensibilidad a los andrógenos" (AIS) -más conocido como "Síndrome de Morris", "Síndrome de feminización testicular" o "pseudohermafroditismo masculino",, "Hiperplasia suprarrenal congénita" (HSC) -"síndrome adrenogenital" o "pseudohermafroditismo femenino"- y casos de hipospadias - abertura de la uretra con una ligera desviación de la punta del pene, en el tronco o en la base de éste.

2 John Money introdujo conceptos como el de "identidad de género" y el de "rol de género": definió la identidad de género como "la afinidad, unidad y persistencia de la individualidad de uno mismo como hombre o mujer, en mayor o menor grado, ya que es experimentada en la conciencia y la conducta. El rol de género es todo lo que una persona dice y hace para indicar al ego el grado en que uno es masculino o femenino (o, raramente, ambivalente); incluye, pero no se restringe, a la excitación y respuesta sexual. La identidad y rol de género son los dos lados de una moneda, y por tanto inseparables. La identidad de género es la experiencia privada del rol de género, y el rol de género es la manifestación pública de la identidad de género" (Gooren, 2004).
} 
En los años sesenta, los avances de la cirugía plástica combinados con esta teoría de "Genitales + Crianza" de la identidad de género de Money, aportaron una racionalización teórica que condujo a los médicos a recomendar la cirugía "correctiva" en muchos tipos de recién nacidos intersexo. La idea era que, una vez asignado médicamente el sexo del bebé, hacer que los genitales se vieran estéticamente correctos -ya fuera de niño o de niña-, y luego criarle en su correspondiente género, creyendo que crecería con la identidad de género asignada. Sin embargo, afirmaban que los dos años era la edad crítica en la cual se fijaba la identidad de género, sin posible vuelta atrás. El caso tratado por Money que monopolizó durante varias décadas la literatura médica sobre intersexualidad fue el conocido como caso John/Joan ${ }^{3}$. Este experimento sentó precedente para la realización de reasignaciones de sexo como tratamiento estándar en miles de recién nacidos con genitales "anómalos". Su planteamiento liberaba, al menos en teoría, a los sujetos inmersos en la dificultad de llevar vidas de hombres y de mujeres sin poseer las "especificaciones biológicas" de su sexo.

A pesar de que en la actualidad los protocolos que John Money desarrolló son duramente criticados por el estamento médico, comprobaremos que paradójicamente, los discursos y protocolos actuales en España para situaciones intersexuales no se alejan tanto de sus orígenes. A día de hoy, los protocolos que se siguen en España para decidir el sexo definitivo que se asignará a un recién nacido intersexual responden, en mayor o menor grado ${ }^{4}$, a diferentes criterios: se considera la anatomía de los genitales externos, el cariotipo, el tipo de gónadas que presente -esto marcaría la capacidad reproductora futura-, los resultados endocrinológicos o respuesta hormonal, la capacidad funcional, las posibilidades de reconstrucción quirúrgica y la decisión o expectativas de los padres; aunque en última instancia, el propio criterio del profesional médico que está a cargo de esa persona, y dependiendo de la experiencia que tenga en situaciones similares, inclinará la balanza hacia lo "femenino" o hacia lo "masculino".

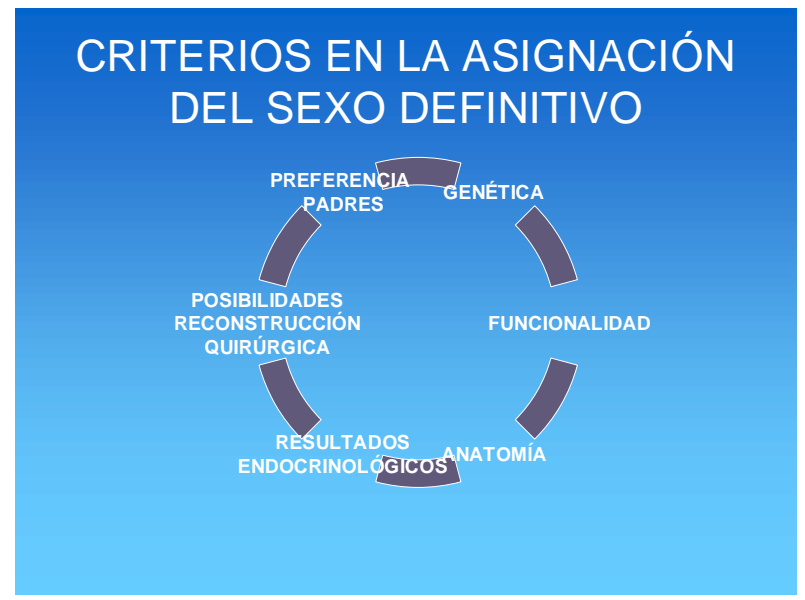

IMAGEN 1. Esquema con los criterios en la asignación de sexo definitivo en bebés intersexo (Elaboración propia).

\footnotetext{
${ }^{3}$ Ver John Colapinto (2000). As nature made him. The boy who was raised as a girl. La historia se puede encontrar colgada en la red en castellano en la página de la Fundación para la Identidad de Género: http://figinternet.org/mod-subjects-printpagepagid-65-scope-all.html (Consultada el 10 de Enero de 2006).

${ }^{4}$ El seguimiento de unos protocolos estandarizados depende en gran medida de la existencia de una infraestructura que permita la creación de equipos multidisciplinares, que disponga de especialistas con experiencia en el diagnóstico y tratamiento de la intersexualidad o de que se cuente con el dispositivo tecnológico necesario para realizar determinadas pruebas diagnósticas e intervenciones quirúrgicas. Evidentemente, esto no es posible en hospitales pequeños de ámbito comarcal.
} 
A continuación nos detendremos en algunos de estos criterios con el fin analizar las asunciones sobre el sexo, el género o la sexualidad que subyacen en determinados discursos y prácticas médicas en el proceso de asignación de sexo en bebés intersexuales.

\title{
1. "El tamaño importa": Sobre dimensiones y placeres
}

"I remember laying in bed late one night after my partner and I made love, my clitoris still feeling the warmth and wetness of the inside of her vagina. It felt good. But I wondered, "Am I the only woman who has done this? Am I the only woman whose clitoris knows the feeling of penetration?"

Kim (1999). "As is", en Dreger (1999)

La anatomía o la morfología genital será el marcador primero y principal para decidir el sexo futuro del recién nacido. Son muchos los esfuerzos dedicados a definir lo que se considera normal o anormal en relación a los genitales externos, así como enorme es el abanico terminológico confeccionado para concretarlo. Pero, ¿qué forma y tamaño deben tener los genitales externos de un recién nacido para que sean considerados clítoris o por el contrario, pene?, ¿qué dimensiones debe albergar una vagina para que no se plantee una reconstrucción de la misma?

Por lo que se refiere a la forma, una estructura fálica precisa estar surcada por una uretra para que sea tipificada como pene y un clítoris, "para que no haya ninguna duda tiene que ser un clítoris que no se vea" (Entrevista a AS, Mayo de 2005). En cuanto al tamaño, son escasos y muy difusos los estudios sobre el tamaño normal de los genitales en un recién nacido, por lo que los criterios en los que se basan para actuar acaban fundamentándose en muchas ocasiones en la propia experiencia profesional:

\begin{abstract}
A pesar de todo, uno dice, con todos los errores, que un pene de un niño debe medir $4 \mathrm{~cm}$. medido como dios manda, como media. Un recién nacido a término son 3.5, más dos desviaciones estándar, que son más 0.8. Pues ya tienes el 4. Yo, para que los residentes se acuerden digo: Mirad, un pito que mide menos de 4, iojo! Si un pito mide de 4 para arriba tranquilidad, que en principio no tiene que tener nada. (Entrevista a PE, Junio de 2005).
\end{abstract}

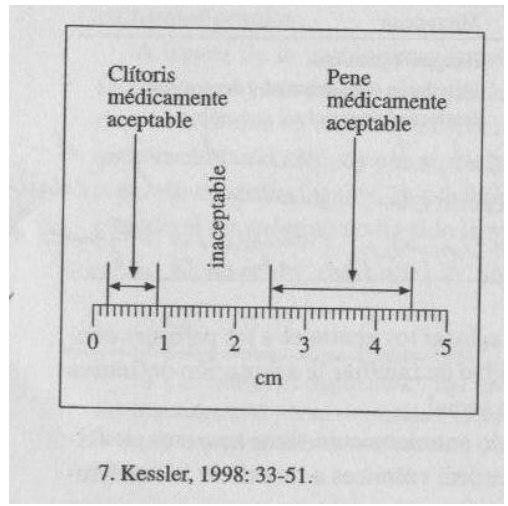

IMAGEN 2. Esquema con las medidas aceptables médicamente para penes y clítoris (Kessler; 1998).

Si bien es cierto, que comparando los estudios existentes con los estándares sobre las medidas y morfologías normales de pene y testículos con los estudios sobre clítoris y labios, son más escasos y mucho más recientes estos últimos. En este sentido, la psicóloga social Suzanne Kessler, en su libro Lessons from the intersexed (1998) hace referencia al androcentrismo en el diagnóstico y tratamiento a intersexuales y plantea además hasta qué punto el hecho de que comiencen a estandarizarse estas 
medidas tiene un efecto contraproducente al inducir a aplicar cirugía "normalizadora" donde antes no se hacía. La autora también analiza la terminología y los presupuestos médicos en cuanto a la forma y el tamaño de los genitales, elaborando una gráfica en la cual presenta las medidas consideradas "normales" médicamente para penes y clítoris, así como aquellas medidas que entran dentro de un espacio de indefinición y que por lo tanto, hacen necesaria la intervención quirúrgica.

También existen criterios para evaluar los tamaños de la vagina, que se relacionan tal y como avanzaba Weeks (1993), con significados culturales sobre el grado de satisfacción sexual. El ideal sobre tamaños de vaginas habla de "vaginas que abrazan penes" (Entrevista a AM, Febrero de 2005) y de lugares estratégicos donde se sitúa el famoso punto $G$ en la mujer. Según este presupuesto, el orgasmo femenino sólo se podría alcanzar a través del punto $\mathrm{G}$ que está situado al final de la vagina. Muy lejos de lo que la naturaleza nos presenta, médicamente existen pocas posibilidades morfológicas para hablar de genitales "normales". Sin embargo, es abundante la terminología elaborada desde la biomedicina para hablar de alteraciones o anormalidades genitales. Por lo que respecta a esta abrumadora terminología referida a la morfología genital nos podríamos cuestionar: ¿De qué hablan las historias clínicas y los manuales médicos sobre estados intersexuales cuando utilizan términos como "megaclítoris", "micropene", "hipertrofía de clítoris", "microfalo", "cuerpos cavernosos hipertrofiados", "pseudopene", "situación cliteroidea de $2 \mathrm{~cm}$ ", "situación peneana de 4cm" o "clítoris peniforme"? Aluden a ¿clítoris grandes en personas $X X$ ? ¿penes pequeños en personas $\mathrm{XY}$ ? ¿Cómo nombrar a quien tiene un cariotipo XXXY?

En realidad, cuando utilizan toda esta extensa terminología, se puede estar hablando de la misma persona, pero en momentos distintos del proceso de sexuación -diagnóstico o tratamiento. Así por ejemplo, pueden utilizar el término "micropene" al analizar los genitales en el momento del diagnóstico -cuando se sabe que su cariotipo es $\mathrm{XY}$-; pero una vez se haya tomado una determinación en cuanto al sexo que se le va a asignar a ese recién nacido, si la decisión es asignarla como niña, el micropene pasará a llamarse a partir de ese momento clítoris hipertrofiado (Fausto-Sterling, 2000).

Esta preocupación por nombrar lo que posteriormente será corregido tecnológicamente, así como cierta obsesión por la apariencia de los genitales externos evidenciada en estos protocolos diagnósticos falométricos, refleja una asociación entre genitales externos e identidad sexual. Es decir, que es imposible conseguir una identidad sexual "correcta" y "saludable" -una supuesta feminidad o masculinidad-, si no se poseen unos genitales externos que respondan a los parámetros establecidos médicamente, y que a su vez responden al estereotipo cultural existente sobre la morfología y aspecto de los genitales externos. Además, y en base a lo dicho, la asignación de sexo fundada en el tamaño y la forma de los genitales se basa, además de en el significado sociocultural de un rasgo físico -el tamaño del pene y el tamaño de la vagina-, en el presupuesto de complementariedad entre sexos, es decir, una sexualidad heterosexual y coitocéntrica. 


\section{Pruebas de "capacidad funcional"}

"Es en el terreno del sexo donde hay que buscar las verdades más secretas y profundas del individuo; es allí donde se descubre mejor lo que somos y lo que nos determina"

Foucault (1985)

Estas pruebas diagnósticas tienen la finalidad de averiguar cual será el futuro comportamiento sexual de esos genitales. Para comprobar si un recién nacido será "funcionalmente sexual" en la edad adulta se realiza una prueba en la cual, a través de la administración de hormonas, se sabrá si un pene en la pubertad (cuando tenga influencia hormonal), tendrá una respuesta sexual y funcional. Esa adecuada "respuesta sexual y funcional" se traduce en un pene con capacidad de erección que pueda "penetrar" hasta alcanzar el fondo de saco de la vagina (lugar donde se presupone la existencia del punto $\mathrm{G}$ en la mujer). En función del resultado de estas pruebas, la decisión definitiva tendrá más o menos posibilidades de dirigirse hacia lo "femenino" o hacia lo "masculino". Con este criterio diagnóstico se repiten los discursos que hablan de un tipo de sexualidad, que no se entiende sin penetración, y de unos presupuestos médicos sobre una sexualidad coitocéntrica y además heterosexual.

\section{Las posibilidades de reconstrucción quirúrgica}

\section{"You cannot remove our existence by removing our anatomy" Morris (2004)}

Las posibilidades tecnológicas serán en muchos casos las que decidirán el sexo definitivo del recién nacido. A pesar de que los avances en cirugía urológica, fundamentalmente desarrollados a partir del tratamiento a personas transexuales, están modificando los criterios de asignación habituales que asignan "por defecto" el sexo femenino a bebés intersexuales, las respuestas no ofrecen titubeos: a día de hoy en España, no existen cirugías correctivas adecuadas para reconstruir penes mal inervados o demasiado pequeños, por tanto, siguen siendo las posibilidades tecnológicas las que establecen el horizonte de lo posible:

\footnotetext{
Si no hay posibilidades de construir un pene funcional se asigna como mujer [...] Si es XY pero nunca vas a poder construir un pene funcional, tendrás que decidirte por asignar hacia el sexo femenino, porque en caso contrario cuando quiera tener relaciones sexuales se frustrará y será realmente dramático y traumático. Lo mismo si es al revés y no puedes conseguir una hembra funcional (Entrevista a AS, Mayo de 2005).
}

A pesar de que actualmente se trabaja en el perfeccionamiento de técnicas quirúrgicas como la faloplastia o la metodoioplastia -o técnica del micropene-, las limitaciones tecnológicas no representan del todo un obstáculo, ya que en el caso de decidir dirigir el desarrollo hacia lo "femenino", se cuenta con poder inestimable de las hormonas que suplirán las limitaciones de la cirugía. 


\title{
Las tecnologías biomédicas en el proceso de asignación de sexo: hormonoterapia y cirugía genital.
}

\author{
“- Interviewer: What causes sex? \\ - Dr. Arika Aiert: Well, soft pink lighting, a glass of wine, "If You Don't Know Me \\ By Now" on the CD, and a nice butch friend who doesn't think I'm a Feminists \\ Traitor because I wear dresses and heels. That usually does it. 'Couse, that's \\ just me. \\ - Interviewer: Oops...I meant what determines sex? \\ - Dr. Arika Aiert: Oh! Surgeons determine sex." \\ Kira Triea (1998) "Interview with Dr. Arika Aiert"
}

El ajuste de cuerpos intersexuales implica no sólo el diagnóstico, sino también intervenciones hormonales y quirúrgicas. Éstas van dirigidas a controlar cuerpos que dejados llevar por su propia naturaleza supondrían una amenaza para unos reducidos esquemas binarios de dos sexos/dos géneros. Ambos tratamientos, cirugía y hormonas, trabajan conjuntamente en el proceso de creación de cuerpos artificialmente sexuados.

La cirugía "correctiva" genital en bebés intersexo va dirigida a "normalizar" genitales que por su apariencia son etiquetados como "ambiguos", "deformes", "anómalos" u "ofensivos", aunque en la mayoría de casos no sean en sí mismos dolorosos ni dañinos para la salud física. Los argumentos médicos utilizados para justificar las intervenciones sobre estos genitales intactos son la presión y angustia de los padres, la probabilidad esperada de que el niño sufra daño emocional debido al rechazo social y los problemas jurídico administrativos derivados de esta indefinición ${ }^{5}$.

Los genitales intersexuales son diamantes en bruto para los cirujanos. Con el bisturí pulen esos "micropenes" o esos "clítoris hipertrofiados" hasta conseguir esos cuerpos insumisos de los que hablan Silvia García Dauder y Carmen Romero (en prensa). Algunas de las técnicas quirúrgicas más recurrentes surgidas en las entrevistas fueron la faloplastia -para la "creación" de neopenes-, la clitorectomía -para la reducción de clítoris-, la vaginoplastia -para la "creación" de neovaginas-, y la clitoridoplastia -para la construcción de clítoris. En la actualidad en España, la faloplastia es una técnica que está perfeccionándose pero de la que no se garantizan buenos resultados y por lo tanto no se confía del todo: "Salvo esos casos concretos el hacer un pene que luego sea sexualmente activo es muy complicado, y las pocas veces que lo hemos hecho el funcionamiento ha sido más bien desastroso" (Entrevista a AM, Febrero de 2005).

Ya en 1982, John Money, al describir el Síndrome de Insensibilidad a los Andrógenos (AIS), hablaba de mujeres con cariotipo XY con tenían una vagina en fondo de saco que no poseía la suficiente profundidad como para permitir un coito satisfactorio, y que por lo tanto precisaba ser alargada quirúrgicamente a mediados de la adolescencia o después de ésta. La "construcción" de neovaginas es considerada en la actualidad una de las técnicas más fáciles en cirugía, aunque los logros médicos en esta especialidad concreta van dirigidos a abandonar técnicas más cruentas donde se precisa

\footnotetext{
${ }^{5}$ El tiempo para inscribir a un recién nacido en el registro civil es de un mes después del nacimiento, y no se puede hacer sin asignar un sexo -mujer o varón- al mismo. Del mismo modo ocurre con otro tipo de documentos tales como el DNI o pasaporte: no existe una tercera casilla o más para un tercer sexo o varios. 
bisturí, por otras que podríamos llamar "técnicas de dilatación progresiva". Según explicaciones de los informantes, pudimos saber que algunas de las neovaginas se construyen a partir del colon o de injertos de piel de la propia paciente. Afirmaban que la intervención era muy fácil, pero parece ser que el mantenimiento de esa intervención no lo es tanto, pues como expresaba un médico: "esa cavidad deberá dilatarse periódicamente para mantenerse en condiciones" (Entrevista a PE, Junio de 2005). Tal argumento hace plantearse a los profesionales médicos retrasar la intervención justo hasta el momento de la adolescencia en la cual se cree que la niña empezará a tener apetencias sexuales:

[...] y morfológicamente ha quedado bastante bien. Y luego a partir de ahí, pues ya viene un poco la conexión una vez más con el equipo de cirugía para intentar hacerlas mujeres funcionales. Si efectivamente, como era el caso de esta niña era un $X Y$, no tiene genitales internos de ningún tipo, y claro, no te queda más remedio que hacerle una neovagina. Entonces, en este caso en concreto, me estoy acordando por la última o la penúltima conversación que tuvimos con la madre, estuvimos comentando este caso, la niña ya tiene 12 o 13 años y claro, estuvimos comentando con la madre que hay que hacerla niña funcional, hay que hacerle una vagina funcional [...] Es la madre la que, con las conversaciones que tenga con la hija es cuando deciden cuando hay que hacerle, porque una vez que hayas hecho una neovagina, hay que usarla. Si no, muchas veces se atrofia, o se... Es complicado. Debe mantenerla en condiciones, tienen que usar dilatadores o rollos de estos. Entonces es preferible cuando la niña, digamos por sus apetencias, va a tener relaciones sexuales, es cuando ya se hace la operación; se hace la neovagina para que la use (Entrevista a PE, Junio de 2005).

Así, la intervención quirúrgica -o vaginoplastia- proporcionará un espacio receptor, en primer lugar, para un objeto llamado tallo de Hegar $^{6}$, que se introducirá diariamente en esa cavidad y la mantendrá permeable y dilatada; y en segundo lugar, pasará el relevo a un pene que culminará la función dilatadora. El tallo de Hegar, utilizado en la biomedicina también para el tratamiento de la vaginitis, es una técnica muy valorada por muchos profesionales médicos porque según ellos es inocua, es decir, evita las "incómodas" cirugías y complicaciones de las mismas como infecciones, hemorragias, etc. Todo este proceso, en resumen, presupone evidentemente varias cosas: el momento en el cual comenzará a tener relaciones sexuales; el tipo de relaciones sexuales que va a tener esa niña y la frecuencia con la que tendrá esas relaciones heterosexuales coitales, pues supone que si no es penetrada periódicamente acabará atrofiándose ese tejido. En cualquier caso, la finalidad de estas técnicas es construir una vagina de dimensiones suficientes para crear mujeres funcionales, es decir, mujeres que dispongan de una vagina con capacidad para albergar un pene.

En el caso de que la decisión sobre el sexo definitivo haya sido mujer, y las dimensiones de lo que sería su futuro clítoris se consideran demasiado grandes, éste también es manipulado y en su caso "reducido" y "(re)construido" a gusto del cirujano a través de las técnicas conocidas como clitorectomía y clitoridoplastía. Sin embargo, uno de los informantes me explicaba como estas técnicas no están exentas de riesgos y contradicciones. Por ejemplo, el reducir mucho el clítoris supone eliminar "su órgano sexual de más satisfacción"; pero el no hacerlo ha originado, en muchos casos, que "cuando se hincha en las relaciones sexuales, como no tiene espacio para hacerlo, las chicas se quejan de unos dolores púbicos terribles" (Entrevista a NR, Enero de 2005).

Según lo planteado, las personas que han pasado por los quirófanos para construir penes o clítoris estándar estarán destinados a dormir todas las noches con un pene en el interior, hasta que un pene

\footnotetext{
${ }^{6}$ El tallo de Hegar consiste en unos dilatadores con forma de cilindros metálicos de punta semirroma, con un calibre que va de 1 a 25mm, y con los que a través de la inserción periódica en la vagina, ésta se hace permeable y dilatada.
} 
sustituya al tallo de Hegar; a sufrir unos dolores terribles debido a una praxis imperfecta, o en última instancia -y parece ser que éstas son las más afortunadas-, a hipotecar su clítoris para prevenir posibles complicaciones.

La utilización de estas técnicas quirúrgicas de forma indiscriminada ha sido muy criticada desde diferentes ámbitos, especialmente desde el activismo político intersex surgido entre otras cosas como respuesta a estas prácticas. Así por ejemplo, la directora de la Intersex Society of North America (ISNA), Cheryl Chase, critica duramente estas "correcciones genitales occidentales", tachándolas de "normalización violenta" y poniéndolas al mismo nivel que las ablaciones genitales no occidentales:

Mientras desde el primer mundo se critica a los otros salvajes tradicionales que bajo el pretexto de rituales culturales perpetúan formas de violencia contra los cuerpos y la sexualidad de las mujeres, se olvida que la ciencia occidental también genera rituales culturales que incitan y promueven violencias corporeizadas con iguales pretensiones de producir individuos socio-sexualmente ajustados: «"su" amputación de genitales es un ritual bárbaro; el "nuestro" es científico. El suyo desfigura; el nuestro normaliza lo desviado» (Chase, 2005).

Complementariamente o de forma independiente a la cirugía, las hormonas trabajan moldeando ese cuerpo que será mostrado públicamente, y como decía Moisés Martínez (2005) refiriéndose al tratamiento en casos de transexualismo, "ellas son las verdaderas responsables de la transformación física". Para casos de intersexo, las hormonas serán la vigilancia más eficaz sobre unos cuerpos que conforme se acercan a la adolescencia podrían resultar peligrosos para el mantenimiento del orden sexuado. Se argumenta cómo el efecto de las hormonas sobre los caracteres sexuales secundarios ${ }^{7}$ eliminará cualquier signo de ambigüedad y por lo tanto garantizará una identidad de género futura acorde con el sexo decidido al nacer; siendo éstas, el mejor seguro de vida para una identidad sexuada "única" y "verdadera". Así, el poder de las hormonas se sobreestima de tal forma que se presupone que conseguirán una identidad de género acorde con los genitales creados:

Casi siempre lo hemos hecho una niña, independientemente del cariotipo, ya te digo, el caso que ahora tenemos es un $X Y[\ldots]$ Y a partir de ahí lo que hacemos es empezar a dar pequeñas dosis de estrógenos, sensibilizar el estado general del resto del organismo con discretos niveles de hormonas femeninas y cuando llega, se acerca la pubertad -incluso creo que tengo fotos de uno de estos casos, es una, fenotípicamente, morfológicamente es una chica, y además guapa. Quiero decir, que es rubia, pelo largo y tal. Quizá luego te encuentras con una estructura, un poquito, un poquito esquelética, masculina, pues son niñas con hombros un poco anchos pero que pasan completamente desapercibidas.- $Y$ es en el momento de la pubertad en la que cargamos la mano en la dosis de estrógenos, generalmente si podemos, 17b estradiol que es la hormona femenina fisiológica, y si no, pues con lo que puedes, pues el 17b estradiol no está comercializado en España (Entrevista a FA, Noviembre de 2004).

La hormonoterapia, es decir, la administración de hormonas en dosis cada vez más altas es la baza tecnológica con la que cuenta la biomedicina para evitar sorpresas inesperadas. Al hablar de estas sorpresas inesperadas, nos referimos a lo que un informante llamaba "la madre del cordero" (Entrevista a AS, Mayo de 2005) cuando llega la pubertad; ya que después de haber asignado un sexo determinado, las gónadas pueden entrar en funcionamiento y dirigir el desarrollo físico hacía el sexo contrario al asignado y esperado. Los efectos de la química hormonal se sobrevaloran en tal medida, que tal y como leemos en el fragmento de entrevista anterior, pueden "crear" no sólo mujeres, en este caso, sino que además mujeres que representan ideales de feminidad en nuestra sociedad: "guapa, rubia, de pelo largo". De este modo las hormonas se convierten en una fuente

\footnotetext{
${ }^{7}$ En el caso de las hormonas masculinas producirán modificaciones en el timbre de voz, en el desarrollo y la distribución del pelo, desarrollo de la musculatura, crecimiento de la laringe o alargamiento del clítoris. Por lo que respecta a las hormonas femeninas provocarán, entre otras cosas, un crecimiento mamario y una redistribución de la grasa.
} 
constante de vigilancia de la identidad sexual "verdadera", aunque para que éstas no sufran en su trabajo ningún sabotaje, los protocolos exigen la extirpación de todo el tejido gonadal que no corresponda con el sexo asignado. Por ello, una de las normas básicas después de decidirse por un sexo u otro, es: "eliminar quirúrgicamente los restos de tejido gonadal del otro sexo -excepto en el síndrome de Morris $^{8}$ en el que no es aconsejable" (Entrevista a FA, Noviembre de 2004). Estas cirugías tienen el pretexto de borrar toda huella posible del otro sexo, que con la pubertad pudiera empezar a actuar y romper esa supuesta identidad de género estable que de forma ideal se habría conseguido: "nos es muy fácil dar estrógenos, es muy fácil. Entonces, quitas las gónadas, vas haciendo una pequeña impregnación a partir de los 5-6 años y cuando llega la pubertad, le metes estrógenos a toda caña y ya está" (Entrevista a FA, Noviembre de 2004).

Para los médicos, el daño emocional producto del rechazo social que pudiera ocasionar el poseer una apariencia corporal sexuada poco habitual o una anatomía inusual es mucho mayor que el posible daño físico derivado de efectos iatrogénicos de la cirugía plástica, así como los efectos secundarios del tratamiento hormonal. Como Kessler (1998) explica, la intervención médica está justificada a favor de producir individuos socialmente ajustados: "un problema estético-social se «cura» médicamente". Según esto, la labor médica tiene como fin responder más a unas necesidades sociales que realmente a lo que es la patología en sí, y por ello las decisiones y acciones tomadas no son cuestionadas y además son reconocidas, entrando decididamente en la esfera de lo sagrado.

\section{"Errores" en el proceso de asignación de sexo}

"But my good woman, you are a man". Alice Dreger, (1998)

Tal como avanzábamos en apartados anteriores, según los postulados de Money, la identidad de género se fija antes de los dos años. Por lo tanto, y siguiendo este modelo, los genitales deberían modelarse quirúrgicamente antes de los 18 meses en recién nacidos intersexuales, ya que el aspecto de los genitales se relaciona directamente con el grado de ajuste social-sexual. De las respuestas ofrecidas por los informantes se extrae que sitúan el momento de la primera intervención quirúrgica, si es posible, sobre el primer año de vida, aunque de forma más general, antes de su ingreso institucional, es decir, cuando tengan que empezar el colegio y se vean los genitales con otros niños. En cualquier caso, se considera que "es algo que no puede demorar" (Entrevista a NR, Enero de 2005):

Hay que definirlo antes de que fije el sexo psicológico. ¿Cuándo fija el sexo psicológico? Meses de vida. Un chico se empieza a identificar como chico o como chica, por lo tanto ya es el momento. Segundo, otro momento importante: cuando empieza a ir a la escuela. Cuando empieza a ir a la escuela él se mira, y hace pis como chica en su orinal o como chico hace de pie cuando tiene un año o dos. Bueno, ya tiene que saber cómo es: y si hace un pis como varón empiezan a ir las cosas mal, y si hace pis de pie como chica también las cosas empiezan a ir mal. Son pequeñas chorradas, pero si conviene dejar claras desde el principio: que el chico tenga una buena identificación sexual (Entrevista a NR, Enero de 2005).

\footnotetext{
${ }^{8}$ Hay argumento médicos que desaconsejan extirpar las gónadas en personas con Síndrome de Insensibilidad a los Andrógenos (AIS) porque según algunos autores en la pubertad hay una conversión de hormona femenina que resulta interesante desde el punto de vista funcional.
} 
Esta premura a la hora de "normalizar" los genitales externos entra en contradicción cuando los profesionales médicos entrevistados plantean las dificultades con las que se encuentran para que la persona se identifique física y psíquicamente con el sexo asignado. En su discurso hay un factor que por ahora escapa al control médico, concretamente el momento en el cual se fija la identidad sexual en los humanos. Muchos son los estudios e hipótesis que sitúan la formación de una supuesta identidad sexual verdadera y de la orientación sexual -homosexual o heterosexual- en el periodo o vida fetal: "la construcción de la identidad de género es anterior al cuerpo y por lo tanto en la reasignación del sexo pueden haber problemas" (Entrevista a TS, Diciembre de 2004). Este planteamiento, dicen, está haciendo reconsiderar los criterios de facilidad o posibilidades de reconstrucción quirúrgica. Así por ejemplo, Piró Biosca (2002) plantea como ha constatado que la impregnación hormonal del cerebro fetal condiciona la orientación sexual definitiva del individuo. Lo ejemplifica aludiendo a estudios realizados a niñas ${ }^{9}$ diagnosticadas con Hiperplasia Suprarrenal Congénita (HSC) en los cuales argumentan como estas niñas en la infancia tienen un comportamiento similar a los varones de su edad y que en la edad adulta hay un incremento de la bisexualidad o de la homosexualidad en estas pacientes. Otros argumentos relacionan la diferencia entre sexos o la existencia de la homosexualidad con conformaciones neuronales y cerebrales: "tú tienes un hipotálamo distinto al mío. Tú tienes ciclos, porque tu hipotálamo es bifásico [...] Yo tengo un hipotálamo unifásico, no puede ser igual. Otra cosa es que lo podamos demostrar, pero eso no puede ser" (Entrevista a AM, Febrero de 2005).

Todos estos esencialismos que rodean el discurso biomédico referente a las diferencias entre sexos o a la orientación sexual ayudan a los propios profesionales médicos a justificar los "errores" o "equivocaciones" que cometen en el proceso de asignación de sexo en recién nacidos intersexo. Por ejemplo, cuando un profesional médico dice que el resultado de la asignación de sexo fue desastroso, normalmente después de que los padres u otro profesional tomará una decisión diferente a la suya en cuanto a la asignación del sexo de su hijo, se refiere a:

$1^{\circ}$ Que la identidad de género con la cual se identificó esa persona era distinta a la del sexo que se le asignó:

\begin{abstract}
Las llamadas feminización testicular -ya se llaman mal- bueno, normalmente por muchas razones médicas el sexo cromosómico $\mathrm{XY}$, se educan como mujeres, ahí es donde vamos a tener un problema seguro. Decíamos y es verdad, nos lo decían, cuando éramos estudiantes o cuando éramos médicos más jóvenes, decían que la educación era lo fundamental y que por lo tanto educando durante toda la infancia como fémina, como mujer, pues no iban a tener problemas, pero si estamos viendo que los problemas sexuales, y sobre todo estos XY y luego al final aceptan su rol varón. [...] Hombre, se dice que cuando el cerebro se impregna de testosterona que es la hormona que en un momento determinado va a poner en marcha todo el camino de varón, y si falla, es por lo que ya se pasa prácticamente a mujer, genitalmente, en este aspecto de genitales ambiguos, pero por lo visto, una vez impregnado el cerebro de testosterona, ya es, vamos, predomina mucho los síntomas, y eso es lo que estamos ocurriendo por muchas razones (Entrevista a AS, Mayo de 2005).
\end{abstract}

$2^{\circ}$ Que generó una orientación sexual homosexual:

[...]¿Cuánta más homosexualidad hay en un sujeto intersexual? ¿Por qué está marcado? ¿Está marcado por las hormonas intraútero? Un feto femenino con una hiperplasia suprarrenal que ya está lleno de andrógenos intraútero, su hipotálamo también está lleno de andrógenos ¿Da igual que una niña que no está llena de andrógenos? ¿Esa tendencia a la homosexualidad que estas niñas tienen con mayor penetrancia que una mujer normal, estará

\footnotetext{
${ }^{9}$ En este caso hablan de "niñas" porque su cariotipo es XX.
} 
condicionado por la impregnación hipotalámica y cortical del andrógeno fetal? ¿O del andrógeno postfetal? (Entrevista a NR, Enero de 2005).

La ecuación sexo-género-deseo-prácticas sexuales vuelve a dotarse de sentido con este planteamiento biomédico, pues como afirman Romero y García Dauder (en prensa), desde la biomedicina se considera que una identidad de género saludable es aquella que se corresponde con una anatomía externa, independientemente de que haya sido conseguida mediante cirugía y hormonas; como una sexualidad heterosexual coitocéntrica, que en el caso de varones implica poder penetrar y en las mujeres desear ser penetradas. Cuando esta fórmula no reproduce esta esperada coherencia, aparece en boca de los médicos la afirmación de que ha habido un error en la asignación:

Realmente es muy difícil y luego se dan muchos casos que la identidad sexual es errónea y, o se sienten hombres o se sienten atraídos por personas de su mismo sexo, no tienen una identidad sexual clara, pero realmente no se puede saber la cantidad de influencias hormonales durante el periodo de gestación que se tuvo y que actuaron (Entrevista a SL, Mayo de 2004 ).

El periodo fetal queda asumido como el momento crítico y definitorio, que partiendo de una estructura cromosómica en interacción con todo un potente artefacto químico, dispondrá nuestra esencia futura, una esencia a la que siempre estaremos sujetos. La principal paradoja radica en que, gracias a la perspectiva biologicista médica sobre la formación de la identidad sexual durante el periodo intraútero, el aparato biomédico puede eludir cualquier posible responsabilidad en el caso de ser denunciados por error médico en la asignación de sexo en bebés intersexuales. Lo que no es demostrable no es denunciable, y en este caso, los límites de la ciencia aparecen como justificación ante posibles errores.

\title{
Conclusiones: legitimando seres interestructurales
}

\author{
"Muchas veces me pregunto la de vidas que habré cambiado. Que \\ existiera un antes y un después de entrar yo en la habitación y después \\ de salir." \\ Entrevista con SL, 28 de Mayo de 2004.
}

En los discursos biomédicos sobre diagnóstico y tratamiento de bebés intersexuales, conceptos como los de sexo, género, identidad sexual, identidad de género, orientación sexual o prácticas sexuales, se acaban confundiendo. Pero, donde no hay lugar a duda, es que cualquier combinación posible responde a un modelo sexual normativo que presupone una "coherencia con el hecho sexual", es decir, la necesidad/obligatoriedad de poseer un solo sexo biológico que sea coherente con una identidad de género y con un rol de género, y que a su vez genere un deseo heterosexual.

Esta secuencia es el resultado de una serie de presupuestos sobre sexo, género y sexualidad que de forma más o menos explícita nos hemos ido encontrando en el análisis de los discursos y protocolos médicos de asignación de sexo en bebés intersexuales. A modo resumen, esos presupuestos pueden verse en la TABLA 1. 


\begin{tabular}{|l|l|}
\hline PRESOCIAL & $\begin{array}{l}\text { Condición intrínseca, biológicamente } \\
\text { determinada. No es necesario hacer ningún } \\
\text { esfuerzo especial ni consciente para } \\
\text { conseguir niños que se eduquen como } \\
\text { hombres y niñas que se eduquen como } \\
\text { mujeres. }\end{array}$ \\
\hline COMPLEMENTARIEDAD & $\begin{array}{l}\text { El hombre y la mujer son dos polos, no existe } \\
\text { el uno sin el otro, se presuponen el uno al } \\
\text { otro, pero son mutuamente excluyentes. Las } \\
\text { divisiones sociales, laborales o de otro tipo se } \\
\text { consideran complementarias. }\end{array}$ \\
\hline ECUACIÓN SEXUALIDAD=PROCREACIÓN & $\begin{array}{l}\text { La relación entre hombres y mujeres es } \\
\text { crucial para la supervivencia de la especie, } \\
\text { por tanto la diferenciación estará } \\
\text { biológicamente predestinada. Para unos es } \\
\text { considerado un "mandamiento divino" y para } \\
\text { otros como "propiedad inherente". }\end{array}$ \\
\hline ATEMPORALIDAD & $\begin{array}{l}\text { La condición de hombre o mujer en el } \\
\text { nacimiento no está sujeta a cambios. }\end{array}$ \\
\hline ECUACIÓN APARIENCIA & $\begin{array}{l}\text { La masculinidad y feminidad presente y futura } \\
\text { puede deducirse de una inspección } \\
\text { (superficial) de los genitales externos. }\end{array}$ \\
\hline EXTERNOS=IDENTIDAD SEXUAL & \multicolumn{1}{c}{}
\end{tabular}

TABLA 1. Presupuestos médicos sobre sexo/género/sexualidad ${ }^{10}$.

La biomedicina, así como los profesionales médicos que trabajan en ella, se constituyen como el brazo ejecutor de unos mandatos socioculturales que en nuestra sociedad naturalizan la diferencia sexual, focalizándola y definiéndola por sus funciones reproductoras y sus órganos diferenciales. Pero, ¿qué sucede cuando la apariencia genital es distinta al estereotipo que tenemos interiorizado sobre la misma? ¿podemos seguir hablando de mujeres y varones? ¿de dónde surgen las medidas que nos alertan de la diferencia? ¿cómo hablar de heterosexualidad u homosexualidad si no puedes clasificar a alguien como mujer o varón? Y en esta línea, ¿cómo hablar de identidad de género? ¿cómo hablar de masculinidad o feminidad? ¿y de roles de género?

Todas las categorías conocidas que definen el sexo, el género y la sexualidad en nuestra sociedad entran en crisis cuando se trata de hablar de intersexualidad. La existencia "liminar" dentro de este esquema de las personas intersexuales nos obliga a revisar estos conceptos para, en primer lugar, deconstruirlos, y a continuación redefinirlos. Para ello se vuelve imprescindible retomar los clásicos debates sobre la dualidad y polarización entre conceptos como cuerpo/mente, sexo/género, naturaleza/cultura, genética/ambiente o innato/adquirido, los cuales presuponen una "esencia" detrás de cualquier determinante cultural.

Así, si eliminamos el velo de lo patológico que rodea la intersexualidad, considerándola no sólo no patológica, sino saludable, se puede constituir como concepto transgresor en la creación de identidades fluidas, y al mismo tiempo, constituyendo un modelo teórico que desafía límites y estructuras fijas. De este modo, la realidad de las personas que han sido diagnosticadas y tratadas como intersexuales dejaría de ser historias que hablan de silencio, agonía, culpa, vergüenza y

\footnotetext{
${ }^{10}$ Para elaborar este esquema he utilizado el artículo de Louis Gooren (2004). El transexualismo, una forma de intersexo. 
ocultamiento, para constituirse como el lugar de la reflexión, de la contestación, de la resistencia, y en definitiva, del cambio social.

Lo interesante de la intersexualidad es que en la medida en que es útil para argumentar esencialismos, se constituye por otro lado como concepto con un gran potencial deconstructor. Las categorías surgidas desde posiciones de poder -en este caso la institución biomédica-, al cambiar de contexto adquieren nuevos significados, y depende de quien se apropie de ellas, pueden resignificarlas creando fisuras en modelos hasta el momento inamovibles. La categoría médica de los estados intersexuales está siendo reapropiada y resignificada en nuevos espacios, formando parte entre otros de nuevos discursos feministas o de los estudios y movimientos queer, transgénero o intersex. Estas nuevas propuestas cuestionan la supuesta objetividad científica y al mismo tiempo atacan las clásicas asunciones y modelos de género y sexualidad, defendiendo una realidad sexual que de hecho es intersexual.

A pesar de las aparentemente pocas posibilidades de escapar de las estrechas barreras y normas que definen lo normal en relación al cuerpo y al sexo, podemos plantearnos que todo aquello que supone un peligro, que provoca contradicciones y dudas, finalmente supone una esperanza. Una esperanza de abolición de verdades estables y absolutas, y de posibilidades de consolidación de nuevas identidades fluidas y críticas. Sin embargo, son muchas las preguntas que nos dejamos en el camino: ¿Cómo autorrepresentarse? ¿Cómo huir de la fijación de estereotipos? ¿Cómo romper binarismos? ¿Cómo utilizar el lenguaje? ¿Cómo visibilizarse? ¿Cómo ser en el mundo?

\section{Bibliografía}

Argente Oliver, J. (2000). Tratado de endocrinología pediátrica y de la adolescencia. Madrid: Ed. Doyma.

Behrman, R. E. y Vaugham, V. C. (1990). Tratado de pediatría Nelson. Tomo I. Interamericana McGraw-Hill.

Botella Llusiá, J.; Clavero Núñez (1993). Tratado de Ginecología y Obstetricia. Madrid: Díaz de santos.

Botella, J. y Fernández, A. (1998). La evolución de la sexualidad y los estados intersexuales. Madrid: Díaz de santos.

Calaf, J. (2000). Desarrollo gonadal y genital, y sus alteraciones. En Fertilidad y Esterilidad Humanas. Tomo II: Endocrinología, Ginecología y Anticoncepción. Vanrell, J.A et al., Coord. Barcelona: Ed. Masson.

Chase, C. (1998). Surgical Progress Is Not the Answer to Intersexuality. The Journal of Clinical Ethics, 9(4): 385392.

Chase, C. (2005). Hermafroditas con actitud: Cartografiando la emergencia del activismo político intersexual. En El eje del mal es heterosexual. Figuraciones, prácticas y movimientos feministas queer. GTQ (Grupo de Trabajo Queer). Madrid: Traficantes de Sueños. 
Colapinto, J. (2000). As nature made him. The boy who was raised as a girl. New York: Harper Collins Publishers.

De Armiñan, J. (1971). Mi querida señorita. España.

Dreger, A. D. (1998). Hermaphrodites and the Medical Invention of Sex. Cambridge: Hardvard University Press.

Dreger, A. D., Ed. (1999). Intersex in the Age of Ethics. Hagerstown: University Publishing Group.

Fausto-Sterling, A. (2000). Sexing the body. Gender Politics and the contruction of Sexuality. New York: Basic Books.

Foucault, M. (1985). Herculine Barbin llamada Alexina B. Madrid: Revolución.

Foucault, M. (1998) [1975]. Vigilar y castigar. Madrid: Siglo XXI.

García Dauder, S. y Romero, C. (en prensa). Haciendo el sexo verdadero: discursos, protocolos, prácticas y tecnologías socio-médicas en el diagnóstico y tratamiento de «estados intersexuales». En Actas del IX Congreso Nacional de Psicología Social. A Coruña.

Gooren, L. (2004). El transexualismo, una forma de intersexo. Lecturas, 209: 12.

Gracia Bouthelier, R. (2002). Anomalías de la diferenciación genital. En Estados intersexuales e hipogonadismo. Sociedad Española de Endocrinología Pediátrica (7 Curso de Formación de Postgrado). Barcelona: J\&C Ediciones Médicas.

Kessler, S. (1998a). Lessons from the Intersexed. New Jersey: Rutgers University Press.

Marañón, G. (1928). Nuevas ideas sobre el problema de la intersexualidad y sobre la cronología de los sexos. Revista de Occidente, Año VI, No LXVI: 257-294.

Marañón, G. (1930). La evolución de la sexualidad. Los estados intersexuales. Madrid: Ed. Morata.

Martínez, M. (2005). Transexualidad masculina y presiones sociales de sexo. En El eje del mal es heterosexual. Figuraciones, prácticas y movimientos feministas queer. GTQ (Grupo de Trabajo Queer). Madrid: Traficantes de Sueños.

Money, J. (1952). Hermaphroditism: An Inquiry into the Nature of a Human Paradox. Tesis Doctoral, Harvard University Library.

Money, J. y Ehrhardt, A. (1982). Desarrollo de la sexualidad humana (diferenciación y dimorfismo de la identidad de género desde la concepción hasta la madurez). Madrid: Morata.

Piró Biosca, C. (2002). Estados intersexuales: tratamiento quirúrgico. En Estados intersexuales e hipogonadismo. Sociedad Española de Endocrinología Pediátrica ( $7^{\circ}$ Curso de Formación de Postgrado). Barcelona: J\&C Ediciones Médicas. 
Triea, K. (1998). Interview with Dr. Arika Aiert. CHRYSALIS: The Journal of Transgressive Gender Identities (revista electrónica), 12 Intersex Awakening , Vol. 2. №5.

Weeks, J. (1993). El malestar de la sexualidad. Significados, mitos y sexualidades modernas. Madrid: Talasa.

\section{Preguntas de la editora}

1. Su artículo, junto con el presentado por Ángel Moreno y Jose Ignacio Pinchardo, expone como: frente a las primeras aproximaciones feministas, que utilizaron como herramienta analítica la distinción entre el "sexo" -las diferencias biológicas entre hombres y mujeres- y el "género" -las diferencias socialmente construidas; el estudio de los estados intersexuales pone en evidencia que no sólo el "género" es una construcción social, sino que también lo es el "sexo". Concluyen ustedes que esto ha llevado a la crítica y deconstrucción de las categorías "sexuales" desde distintas corrientes feministas y movimientos queer, transgénero e intersex. ¿Podría desarrollar brevemente algunas de estas propuestas teóricas y detallar el papel que la reapropiación de la intersexualidad juega en ellas? ¿Qué diferencias plantea el tratamiento por estos grupos de la intersexualidad y de la transexualidad? ¿De qué manera se visibilizan en el activismo político de estos grupos?

Efectivamente podemos hablar de un nuevo modelo analítico dentro de los denominados estudios de género donde la intersexualidad, etiqueta surgida dentro del discurso biomédico, adquiere protagonismo. Como decías, estas nuevas propuestas teóricas elaboradas dentro de estudios queer o transgénero o como parte del discurso de movimientos o del activismo político intersex o trans, vuelven a cargar las tintas contra esencialismos, discutiendo binarismos y verdades absolutas y reforzando las teorías sobre la construcción social de las categorías. Puede parecer a simple vista que volvemos a retomar viejos debates ya superados, aunque creo que precisamente estamos en un momento en el cual no resulta baladí reforzar un contradiscurso que amortigüe la avalancha de investigaciones biomédicas que insisten en que la esencia de cualquier hecho social se encuentra en genes, conexiones neuronales o estructuras cerebrales. Con estas teorías biomédicas nuestro destino está marcado incluso antes del nacimiento, y en relación al género, las categorías que desde la aparición de los primeros discursos feministas hasta la actualidad se habían tornado más difusas, vuelven a consolidarse como verdades opuestas para volverse más herméticas y en definitiva, más irreales.

Es precisamente en este punto en el cual advertimos la relevancia teórica de una categoría como la de la intersexualidad. A mediados de la década de los noventa empiezan a visibilizarse testimonios similares de personas que en su momento fueron diagnosticadas bajo la insignia de algún síndrome intersexual y que sufrieron los protocolos médicos de asignación de sexo. El activista intersex Mauro Cabral (2005) lo explicaba así:

Hablaban de sucesos ocurridos hacía mucho tiempo, en su infancia. Sucesos que habían marcado sus vidas y también sus cuerpos. En muchos casos eran sus mismas marcas corporales, inexplicables o mal explicadas, las que llevaron a estas personas a preguntarse por su niñez, por el momento en que todo había comenzado [...] Sus cuerpos, identificados en el momento de nacer como «malformados», «ambivalentes» o «indefinidos», habían sido sometidos una y otra vez a procedimientos médicos y sociales de "normalización", a fin de borrar cualquier evidencia 
de lo monstruoso. Su niñez era el relato mismo de esta intervención, cuyo resultado era denunciado repetidamente como una mutilación ${ }^{11}$.

La relevancia y dramatismo de estas experiencias dio lugar al nacimiento de nuevas corrientes de pensamiento, a la creación de un contradiscurso conocido como activismo o movimiento intersex y al desarrollo de una corriente teórica que deconstruye el paradigma biomédico y opta por la creación de una identidad fluida. Así por ejemplo, la acción política de grupos como la Intersex Society of North America, la Organisation Internacional des Intersexués, el Intersex Support Group Internacional, la Intersex Information and Peer Support, o la United Kingdom Intersex Association recoge dos premisas: "visibilización y denuncia". Por un lado, visibilizan a aquellas personas nacidas con una condición intersexual, siendo para muchas de ellas la intersexualidad su identidad, aunque la marca original fuera borrada por la cirugía y porque según ellos nunca se identificaron con el sexo impuesto. Y por otro lado, denuncian las prácticas médicas relacionadas con la intersexualidad, es decir, la medicalización de la ambigüedad anatómica, y acusan a los profesionales médicos del estigma que les impusieron obligándoles a mantener su historia en secreto. A todo lo anteriormente dicho se une todo un dispositivo conceptual creado y elaborado por los propios activistas, dentro y fuera de las asociaciones, que aparte de sacar del anonimato las experiencias de estas personas, permite cuestionar los esencialismos biológicos, el supuesto objetivismo científico o el dimorfismo sexual en Occidente.

La activista norteamericana Cheryl Chase, fundadora en 1993 de la Intersex Society of North America (ISNA) hace hincapié en que su batalla es por aquellas personas que nacen con un cuerpo inusual, no por conseguir un tercer sexo o un solo sexo. Así, el activismo del ISNA persigue, entre otros objetivos, la creación de comités de ética en los hospitales y cambios legislativos que impidan el ejercicio médico sin control. La principal crítica aportada por asociaciones como el ISNA se centra en que a través de estas cirugías cosméticas se daña la función sexual (por retirar tejido conectivo con capacidad sensitiva), disminuyendo la respuesta erotosexual. Añaden además que los resultados de la reconstrucción cosmética no son casi nunca satisfactorios, y reclaman para sí el derecho a la autodeterminación para decidir por ellas mismas, si es que lo desean en algún momento, pasar por una intervención quirúrgica de sus genitales. (Nieto, 2003: 81). Este es un punto de encuentro entre el activismo y la crítica trans e intersex. Las personas transexuales también reclaman el derecho a cambiar de identidad de género sin tener que modificar su cuerpo mediante tecnologías médicas.

En cualquier caso, los trabajos de autoras como Cheryl Chase, Alice Domurat Dreger, Sharon Preves, Suzanne Kessler o Anne Fausto Sterling, intentan, desde diferentes disciplinas, desestigmatizar la intersexualidad analizando la utilización indiscriminada de las tecnologías médicas en la producción de individuos que entren en sociedad como "mujeres y varones normales heterosexuales" y paralelamente haciendo una crítica incisiva al "dimorfismo sexual" y a la "monosexualidad". Al mismo tiempo, procuran alejar la intersexualidad de la idea de enfermedad, considerando la ambigüedad sexual no sólo no patológica, sino saludable. Cabe destacar otras propuestas teóricas como los

\footnotetext{
11 Texto introductorio del seminario "Tecnologías de género: Poéticas y Políticas de la Intersexualidad" impartido en Corrientes (Argentina) en mayo de 2005. Se puede encontrar en la página web: www.rojas.uba.ar (Consultada el 30 de Enero de 2006 ). 
trabajos de la filósofa Judith Butler $(2001,2002,2004)$ quien plantea la necesidad de nuevas formas de inteligibilidad de lo posible y lo vivible que pongan al sistema binario de sexo/género/deseo en disputa:

¿De qué forma tenemos que replantear las limitaciones morfológicas ideales que recaen sobre los seres humanos de modo tal que quienes se alejan de la norma no se vean condenados a una muerte en vida? (Butler, 2001: 20).

En España, José Antonio Nieto (2003) nos interpela preguntando:

¿Por qué no se replantea la sociedad el binario de géneros? ¿Por qué la medicina no reconoce la experiencia antropológica para cerciorarse de que existen sociedades que admiten más de dos géneros? ¿Por qué no se admite el transgénero como un género más, como un tercer género? ¿Por qué las instituciones sociales se sirven siempre de la medicina, cada vez que se presenta la duda ante la «ambigüedad corporal»? ¿Por qué la identidad corporal subjetiva cuando es «transgresora» se patologiza? ¿Por qué no se socializa?" (Nieto, 2003: 79)

En definitiva, se está constituyendo un corpus teórico en contraposición a clásicas categorizaciones binarias excluyentes y en pro de categorías fluidas que huyan de compartimentos estancos. Estas nuevas propuestas hablan de cuerpos, géneros e identidades que pretenden desorientarnos, contrariarnos, ya que se sitúan al límite o fuera de nuestros esquemas mentales y por lo tanto nos obligan a replantearnos toda nuestra existencia. Son cuerpos que mudan en el espacio y el tiempo y a los que nos resulta imposible nombrar si no es haciendo un gran esfuerzo creativo. Estas nuevas categorías nos hacen entrar en crisis, obligándonos a aprender a vivir en la inestabilidad de una existencia que es en realidad inconstante y cambiante.

\section{No parece sencillo realizar una etnografía sobre intersexualidad ¿Podría detallar en mayor medida las especificidades técnico I metodológicas de su investigación, así como las dificultades y opciones tomadas en el acceso al campo y la recogida del material? ¿Cómo se plantea continuar el trabajo etnográfico?}

En realidad ha sido, y sigue siendo un constante ir y venir de preguntas y dudas alrededor de un objeto de estudio que me resulta extremadamente rico pero de gran complejidad: ¿Dónde contextualizar? ¿Cómo acotar la muestra para que sea representativa? ¿Cómo utilizar unas técnicas de investigación y una metodología antropológica con este objeto de estudio? Muchas preguntas a nivel metodológico se han sumado al problema de desinformación que tenía sobre el tema en un principio, por lo que el trabajo preliminar que realicé fue establecer una primera toma de contacto; un trabajo exploratorio que me permitiera acabar formulando más preguntas que generando respuestas. Este planteamiento inicial estuvo justificado por una serie de limitaciones encontradas a priori:

- Inexistencia de trabajos de campo sobre intersexualidad en España, ya sea a nivel de la práctica médica, de los propios afectados o protagonistas, o de los posibles colectivos o asociaciones que se hubieran podido formar alrededor del problema.

- Descentralización de la práctica médica en el diagnóstico y tratamiento de la intersexualidad, y la inexistencia de una especialidad clínica exclusiva en el seguimiento de casos de intersexo. 
- El secretismo y ocultamiento alrededor de un diagnóstico de intersexualidad, tanto desde los propios profesionales médicos -por el conocido como "secreto médico"-, como por parte de las personas diagnosticadas como intersexuales que han vivido la experiencia de asignación médica de sexo y de sus familias.

- Inexistencia de registros formales que recojan casos de asignación médica de sexo en personas intersexuales, o imposibilidad de acceder a los mismos.

- Incidencia baja de este tipo de situaciones, y por lo tanto, dispersión geográfica y dificultad de acceso a informantes.

Estas circunstancias de partida me han obligado a adaptarme a mi objeto de estudio utilizando mis propias redes sociales más cercanas y en definitiva a agotar los recursos que me han dado los informantes. Para acceder a informantes clave en un primer nivel de análisis centrado en el trabajo médico, hubo algunos aspectos que me facilitaron la entrada al campo y otros que la hicieron más difícil. Como facilidades puedo explicitar la posición que ocupo como enfermera trabajando en un hospital, hecho que me ha facilitado por un lado el conocimiento de la estructura y funcionamiento del sistema sanitario; por otro cierta familiaridad con el lenguaje médico; y por último, y el más importante, el acceso a personal sanitario que, a modo de efecto "bola de nieve", me ha facilitado el contacto con otros profesionales. Esta dinámica situación me permitió moverme de un ámbito prácticamente comarcal que se caracterizaba por una desinformación acerca de casos de intersexualidad, a grandes complejos hospitalarios con una larga trayectoria en el diagnóstico y tratamiento de situaciones de este tipo. Puedo decir con esto que la selección de la muestra ha sido más bien "forzosa" y ha respondido en principio a criterios de proximidad física, por tanto, el prescindir del rigor en la selección ha sido más una necesidad que una elección. Los dispositivos metodológicos utilizados han sido la entrevista en profundidad como instrumento para la obtención de datos, aplicando los principios del análisis del discurso para la descripción e interpretación de los mismos.

En cuanto a cómo me planteo continuar con el trabajo etnográfico, al que ha sido el trabajo dentro del ámbito sanitario y con profesionales médicos, se sumarían las experiencias e historias de vida de personas que han sido diagnosticadas con un estado intersexual y que han vivido una asignación de sexo, así como las de sus familiares. Dentro de esta parte del trabajo de campo incluiría a los colectivos o asociaciones dentro de los cuales, de forma más o menos explícita, se incluyen estas personas, analizando cuáles son sus objetivos, propósitos, acciones, etc. De nuevo va siendo las posibilidades de entrar en contacto, a través de redes personales o de asociaciones LGTB la forma de abordar un objeto de estudio que se caracteriza por el secretismo, invisibilidad e incluso el miedo y la vergüenza. En esta nueva etapa contemplaría el uso de internet o de la creación de una página web que garantiza mejor el anonimato. 


\section{Referencias Bibliográficas}

Butler, J. (2001) El género en disputa. El feminismo y la subversión de la identidad. Barcelona: Paidós.

Butler, J. (2002). Cuerpos que importan: Sobre los límites materiales y discursivos del "sexo". Barcelona: Paidós.

Butler, J. (2004) Undoing Gender. New York: Routledge.

Nieto, J. A. (2003). La intersexualidad y los límites del modelo "dos sexos/dos géneros. En Sexualidades. Diversidad y control social. O. Guash y O. Viñuales, Eds. Bellaterra: Barcelona. 